Appl. Set-Valued Anal. Optim. 2 (2020), No. 2, pp. 183-203

Available online at http://asvao.biemdas.com

https://doi.org/10.23952/asvao.2.2020.2.05

\title{
A GENERAL ITERATIVE ALGORITHM FOR GENERALIZED SPLIT FEASIBILITY AND FIXED POINT PROBLEMS
}

\author{
JONG SOO JUNG
}

Department of Mathematics, Dong-A University, Busan 49315, Korea

\begin{abstract}
In this paper, we introduce a general iterative algorithm based on the hybrid steepest descent method for finding a common element of the solution set of generalized split feasibility problem and the fixed point set of a continuous pseudocontractive mapping. We establish the strong convergence of the proposed iterative algorithm and find a minimum-norm element in the common set of the two sets in a Hilbert space.

Keywords. Averaged mapping; Fixed point problem, Generalized split feasibility problem; Minimum-norm point, Pseudocontractive mapping.
\end{abstract}

\section{INTRODUCTION}

Let $H_{1}$ and $H_{2}$ be two real Hilbert spaces. Let $D$ and $Q$ be nonempty convex closed subsets of $H_{1}$ and $H_{2}$, respectively. Let $A: H_{1} \rightarrow H_{2}$ be a bounded linear mapping. Then the split feasibility problem (SFP) is to find a point $z \in H_{1}$ such that $z \in D \cap A^{-1} Q$. In 1994, the SFP was first introduced by Censor and Elfving [7] in finite-dimensional Hilbert spaces for modeling inverse problems, which arise from phase retrievals and in medical image reconstruction. Since then, the SFP has received much attention due to its real applications in signal processing, image reconstruction, with particular progress in intensity-modulated radiation therapy(IMRT), approximation theory, control theory, biomedical engineering, communications and geophysics. We refer to $[3,7]$ and related literatures.

In 2015, Takahashi, Xu and Yao [24] considered the following generalized split feasibility problem (GSFP):

$$
\text { find a point } x^{*} \in H_{1} \text { such that } 0 \in B\left(x^{*}\right) \text {, }
$$

and

$$
y^{*}=A x^{*} \in H_{2} \text { solves } y^{*}=T\left(y^{*}\right),
$$

where $B: H_{1} \rightarrow 2^{H_{1}}$ is a multi-valued maximal monotone mapping, $T: H_{2} \rightarrow H_{2}$ is a nonexpansive mapping and $A: H_{1} \rightarrow H_{2}$ is a bounded linear operator. The GSFP (1.1)-(1.2) includes, as special cases, several split problems, such as the split zero problem (SZP), the split variational inclusion problem (SVIP), the SFP, and split common fixed point problem (SCFPP) $[3,4,7,12,13,18,19]$, which have already been extensively studied and used in practice as a model in the IMRT treatment planning (see $[6,7]$ ) and in many inverse problems arising

E-mail address: jungjs@dau.ac.kr.

Received May 23, 2020; Accepted June 6, 2020.

(C)2020 Applied Set-Valued Analysis and Optimization 
for phase retrieval and other real-world problem; for instance, in computerized tomography, in sensor networks and date computation (see [2, 8, 25]).

A fixed point problem (FPP) is to find a fixed point $z$ of a nonlinear mapping $T$ with property:

$$
z \in C, T z=z \text {. }
$$

In particular, in order to study the GSFP (1.1)-(1.2) coupled with fixed point problem (1.3), Takahashi, Xu and Yao [24] proposed the following iterative method for a point $x^{*} \in F i x(U) \cap$ $B^{-1} 0 \cap A^{-1}($ Fix $(S))$ as follows:

$$
\left\{\begin{aligned}
z_{n} & =J_{\lambda_{n}}^{B}\left(I+\lambda_{n} A^{*}(S-I) A x_{n}\right) \\
x_{n+1} & =\beta_{n} x_{n}+\left(1-\beta_{n}\right) U z_{n}, n \geq 1,
\end{aligned}\right.
$$

where $B: H_{1} \rightarrow 2^{H_{1}}$ is a maximal monotone mapping, $J_{\lambda}^{B}=(I+\lambda B)^{-1}$ is the resolvent of $B, A^{*}$ is the adjoint of $A, U: H_{1} \rightarrow H_{1}$ ia a generalized hybrid mapping and $S: H_{2} \rightarrow H_{2}$ is a nonexpansive mapping. They established weak convergence of the sequence generated by proposed iterative algorithm (1.4).

As we know, Kocourek, Takahashi and Yao [15] first introduced a class of generalized hybrid mappings containing nonexpansive mappings, nonspreading mappings $[16,17]$ and hybrid mappings [23] in a Hilbert space. However, the class of generalized hybrid mappings does not contain generally the class of pseudocontractive mappings. So, the following problems arise naturally:

Question 1.1. Can we replace the generalized hybrid mapping in [24] by the pseudocontractive mapping?

Question 1.2. Can we extend the class of nonexpansive mappings in [24] to the more general class of pseudocontractive mappings?

Question 1.3. Can we establish strong convergence for GSFP (1.1)-(1.2) and the FPP (1.3) instead of weak convergence in [24]?

In this paper, in order to give an affirmative answer to the above three questions, we introduce a general iterative algorithm based on the hybrid steepest descent method for finding a common solution of the GSFP (1.1)-(1.2) and the FPP (1.3) for a continuous pseudocontractive mapping. Then we establish strong convergence of the sequence generated by the proposed iterative algorithm to a common element of the two solution sets, which is a solution of a certain variational inequality. As a direct consequence, we find the unique minimum-norm element of the common set of the two solution sets.

\section{PRELIMINARIES AND LEMMAS}

Let $H$ be a real Hilbert space with the inner product $\langle\cdot, \cdot\rangle$ and the induced norm $\|\cdot\|$, and let $C$ be a nonempty convex closed subset of $H$. A mapping $A$ of $C$ into $H$ is said to be monotone if

$$
\langle x-y, A x-A y\rangle \geq 0, \forall x, y \in C .
$$

A mapping $A$ of $C$ into $H$ is called $\alpha$-inverse-strongly monotone (or $\alpha$-ism) (see [11]) if there exists a positive real number $\alpha$ such that

$$
\langle x-y, A x-A y\rangle \geq \alpha\|A x-A y\|^{2}, \quad \forall x, y \in C .
$$


Clearly, the class of monotone mappings includes the class of $\alpha$-inverse-strongly monotone mappings .

A mapping $T$ of $C$ into $H$ is said to be pseudocontractive if

$$
\|T x-T y\|^{2} \leq\|x-y\|^{2}+\|(I-T) x-(I-T) y\|^{2}, \quad \forall x, y \in C,
$$

and $T$ is said to be $k$-strictly pseudocontractive (see [5]) if there exists a constant $k \in[0,1)$ such that

$$
\|T x-T y\|^{2} \leq\|x-y\|^{2}+k\|(I-T) x-(I-T) y\|^{2}, \quad \forall x, y \in C,
$$

where $I$ is the identity mapping. Note that the class of $k$-strictly pseudocontractive mappings includes the class of nonexpansive mappings as a subclass. That is, $T$ is nonexpansive (i.e., $\|T x-T y\| \leq\|x-y\|, \forall x, y \in C)$ if and only if $T$ is 0 -strictly pseudocontractive. Clearly, the class of pseudocontractive mappings includes the class of strictly pseudocontractive mappings and the class of nonexpansive mappings as a subclass. Moreover, this inclusion is strict (see Example 5.7.1 and Example 5.7.2 in [1]).

Let $B$ be a mapping of $H$ into $2^{H}$. The effective domain of $B$ is denoted by $\operatorname{dom}(B)$, that is, $\operatorname{dom}(B)=\{x \in H: B x \neq \emptyset\}$. A set-valued mapping $B$ is said to be a monotone operator on $H$ if $\langle x-y, u-v\rangle \geq 0$ for all $x, y \in \operatorname{dom}(B), u \in B x$, and $v \in B y$. A monotone operator $B$ on $H$ is said to be maximal if its graph is not properly contained in the graph of any other monotone operator on $H$. For a maximal monotone operator $B$ on $H$ and $\lambda>0$, we may define a singlevalued operator $J_{\lambda}^{B}=(I+\lambda B)^{-1}: H \rightarrow \operatorname{dom}(B)$, which is called the resolvent of $B$. Let $B$ be a maximal monotone operator on $H$ and let $B^{-1} 0=\{x \in H: 0 \in B x\}$. It is well-known that $B^{-1} 0=F i x\left(J_{\lambda}^{B}\right)$ for all $\lambda>0$ is closed and convex and the resolvent $J_{\lambda}^{B}$ is firmly nonexpansive, that is,

$$
\left\|J_{\lambda}^{B} x-J_{\lambda}^{B} y\right\|^{2} \leq\left\langle x-y, J_{\lambda}^{B} x-J_{\lambda}^{B} y\right\rangle, \quad \forall x, y \in H .
$$

The following resolvent identity

$$
J_{\lambda}^{B} x=J_{\mu}^{B}\left(\frac{\mu}{\lambda} x+\left(1-\frac{\mu}{\lambda}\right) J_{\lambda}^{B} x\right)
$$

holds for all $\lambda, \mu>0$ and $x \in H([1,22])$.

In a real Hilbert space $H$, the following equalities hold:

$$
\|x-y\|^{2}=\|x\|^{2}+\|y\|^{2}-2\langle x, y\rangle
$$

and

$$
\|\alpha x+\beta y\|^{2}=\alpha\|x\|^{2}+\beta\|y\|^{2}-\alpha \beta\|x-y\|^{2} \leq \alpha\|x\|^{2}+\beta\|y\|^{2},
$$

for all $x, y \in H$ and $\alpha, \beta \in(0,1)$ with $\alpha+\beta=1$. For every point $x \in H$, there exists a unique nearest point in $C$, denoted by $P_{C} x$, such that

$$
\left\|x-P_{C} x\right\|=\inf \{\|x-y\|: y \in C\} .
$$

$P_{C}$ is called the metric projection of $H$ onto $C$. It is well known [22] that $P_{C}$ is nonexpansive and $P_{C}$ is characterized by the property

$$
u=P_{C} x \Longleftrightarrow\langle x-u, u-y\rangle \geq 0, \quad \forall x \in H, y \in C .
$$


It is also well known [9, Theorem 3.1] that every nonexpansive mapping $T: H \rightarrow H$ satisfies, for all $(x, y) \in H \times H$, the inequality

$$
\langle(x-T x)-(y-T y), T y-T x\rangle \leq \frac{1}{2}\|(T x-x)-(T y-y)\|^{2},
$$

and hence, we get, for all $(x, y) \in H \times F i x(T)$,

$$
\langle x-T x, y-T x\rangle \leq \frac{1}{2}\|T x-x\|^{2} .
$$

A mapping $T: H \rightarrow H$ is said to be averaged if it can be written as the average of the identity $I$ and a nonexpansive mapping, that is,

$$
T=(1-\alpha) I+\alpha S,
$$

where $\alpha$ is a number in $(0,1)$ and $S: H \rightarrow H$ is nonexpansive. More precisely, when (2.7) holds, we say that $T$ is $\alpha$-averaged.

We note that averaged mappings are nonexpansive. Further firmly nonexpansive mappings (in particular, projections and resolvents of maximal monotone operators) are averaged.

We collect some basic properties of averaged mappings and inverse strongly monotone mappings, see, e.g., $[3,19]$.

Proposition 2.1. (i) If $T=(1-\alpha) S+\alpha V$, where $S: H \rightarrow H$ is averaged, $V: H \rightarrow H$ is nonexpansive and $\alpha \in(0,1)$, then $T$ is averaged.

(ii) The composite of finitely many averaged mappings is averaged.

(iii) If the mappings $\left\{T_{i}\right\}_{i=1}^{N}$ are averaged and have a nonempty common fixed point, then

$$
\bigcap_{i=1}^{N} \operatorname{Fix}\left(T_{i}\right)=\operatorname{Fix}\left(T_{1} \cdots T_{N}\right) .
$$

(iv) A mapping $T: H \rightarrow H$ is nonexpansive if and only if $I-T$ is $\frac{1}{2}-i s m$

(v) If $A$ is $v$-ism, then, for $\gamma>0, \gamma A$ is $\frac{v}{\gamma}$-ism.

(vi) $T$ is averaged if and only if the complement $I-T$ is $v$-ism for some $v>\frac{1}{2}$. Indeed, for $\alpha \in(0,1), T$ is $\alpha$-averaged if and only if $I-T$ is $\frac{1}{2 \alpha}$-ism.

The following lemmas were given in [24].

Lemma 2.1. [24] Let $H_{1}$ and $H_{2}$ be real Hilbert spaces. Let $A: H_{1} \rightarrow H_{2}$ be a bounded linear operator such that $A \neq 0$ and let $A^{*}$ be the adjoint of $A$. Let $L$ is the spectral radius of the operator $A^{*} A$. Let $\mathrm{T}: \mathrm{H}_{2} \rightarrow \mathrm{H}_{2}$ be a nonexpansive mapping. Then

(i) $\eta A^{*}(I-T) A$ is $\frac{1}{2 \eta L}$-ism,

(ii) for $\eta \in\left(0, \frac{1}{L}\right)$,

(iia) $I+\eta A^{*}(T-I) A$ is $\eta L$-averaged;

(iib) $J_{\lambda}^{B}\left(I+\eta A^{*}(T-I) A\right)$ is $\frac{1+\eta L}{2}$-averaged.

Lemma 2.2. [24] Let $H_{1}$ and $H_{2}$ be real Hilbert spaces. Let $B: H_{1} \rightarrow 2^{H_{1}}$ be a maximal monotone operator and let $J_{\lambda}^{B}=(I+\lambda B)^{-1}$ be the resolvent of $B$ for $\lambda>0$. Let $A: H_{1} \rightarrow H_{2}$ be a bounded linear operator such that $A \neq 0$ and let $A^{*}$ be the adjoint of $A$. Let $T: H_{2} \rightarrow H_{2}$ be a nonexpansive mapping. Suppose that $B^{-1} 0 \cap A^{-1}(F i x(T)) \neq \emptyset$. Let $\lambda, \eta>0$ and $z \in H_{1}$. Then the following are equivalent: 

(i) $z=J_{\lambda}^{B}\left(I+\eta A^{*}(T-I) A\right) z$;
(ii) $0 \in-A^{*}(T-I) A z+B z$;
(iii) $z \in B^{-1} 0 \cap A^{-1}(F i x(T))$.

Consequently, Fix $\left(J_{\lambda}^{B}\left(I+\eta A^{*}(T-I) A\right)\right)=\left(-A^{*}(T-I) A+B\right)^{-1} 0=B^{-1} 0 \cap A^{-1}(F i x(T))$. Moreover, if $0 \in-A^{*}(T-I) A u+B u$ and $0 \in-A^{*}(T-I) A v+B v$, then $A^{*}(T-I) A u=A^{*}(T-$ I) $A v$ and $\left(-A^{*}(T-I) A+B\right)^{-1} 0$ is convex and closed.

We need the following lemmas for the proof of our main results.

Lemma 2.3. [1, 22] In a real Hilbert space $H$, the following inequality holds:

$$
\|x+y\|^{2} \leq\|x\|^{2}+2\langle y, x+y\rangle, \quad \forall x, y \in H .
$$

Lemma 2.4. [21] Let $\left\{x_{n}\right\}$ and $\left\{z_{n}\right\}$ be bounded sequences in a real Banach space E, and let $\left\{\gamma_{n}\right\}$ be a sequence in $[0,1]$, which satisfies the following condition:

$$
0<\liminf _{n \rightarrow \infty} \gamma_{n} \leq \limsup _{n \rightarrow \infty} \gamma_{n}<1
$$

Suppose that $x_{n+1}=\gamma_{n} x_{n}+\left(1-\gamma_{n}\right) z_{n}$ for all $n \geq 1$ and

$$
\limsup _{n \rightarrow \infty}\left(\left\|z_{n+1}-z_{n}\right\|-\left\|x_{n+1}-x_{n}\right\|\right) \leq 0 .
$$

Then $\lim _{n \rightarrow \infty}\left\|z_{n}-x_{n}\right\|=0$.

Lemma 2.5. [26] Let $\left\{s_{n}\right\}$ be a sequence of nonnegative real numbers satisfying

$$
s_{n+1} \leq\left(1-\xi_{n}\right) s_{n}+\xi_{n} \delta_{n}, \quad \forall n \geq 1,
$$

where $\{\xi\}$ and $\left\{\delta_{n}\right\}$ satisfy the following conditions:

(i) $\left\{\xi_{n}\right\} \subset[0,1]$ and $\sum_{n=1}^{\infty} \xi_{n}=\infty$;

(ii) $\limsup _{n \rightarrow \infty} \delta_{n} \leq 0$ or $\sum_{n=1}^{\infty} \xi_{n}\left|\delta_{n}\right|<\infty$.

Then $\lim _{n \rightarrow \infty} s_{n}=0$.

Lemma 2.6. [28] Let $C$ be a closed convex subset of a real Hilbert space H. Let $T: C \rightarrow H$ be a continuous pseudocontractive mapping. Then, for $r>0$ and $x \in H$, there exists $z \in C$ such that

$$
\langle y-z, T z\rangle-\frac{1}{r}\langle y-z,(1+r) z-x\rangle \leq 0, \quad \forall y \in C .
$$

For $r>0$ and $x \in H$, define $T_{r}: H \rightarrow C$ by

$$
T_{r} x=\left\{z \in C:\langle y-z, T z\rangle-\frac{1}{r}\langle y-z,(1+r) z-x\rangle \leq 0, \quad \forall y \in C\right\} .
$$

Then the following hold:

(i) $T_{r}$ is single-valued;

(ii) $T_{r}$ is firmly nonexpansive, that is,

$$
\left\|T_{r} x-T_{r} y\right\|^{2} \leq\left\langle x-y, T_{r} x-T_{r} y\right\rangle, \quad \forall x, y \in H ;
$$

(iii) $\operatorname{Fix}\left(T_{r}\right)=\operatorname{Fix}(T)$;

(iv) Fix $(T)$ is a closed convex subset of $C$.

The following lemmas can be easily obtained (see [27]). We, therefore, omit their proofs. 
Lemma 2.7. Let $H$ be a real Hilbert space. Let $V: H \rightarrow H$ be an l-Lipschitzian mapping with a constant $l \geq 0$, and let $G: H \rightarrow H$ be a $\kappa$-Lipschitzian and $\eta$-strongly monotone mapping with constants $\kappa, \eta>0$. Then for $0 \leq \gamma l<\mu \eta$,

$$
\langle(\mu G-\gamma V) x-(\mu G-\gamma V) y, x-y\rangle \geq(\mu \eta-\gamma l)\|x-y\|^{2}, \quad \forall x, y \in H .
$$

That is, $\mu G-\gamma V$ is strongly monotone with constant $\mu \eta-\gamma l$.

Lemma 2.8. Let $H$ be a real Hilbert space $H$. Let $G: H \rightarrow H$ be a $\kappa$-Lipschitzian and $\eta$ strongly monotone mapping with constants $\kappa>0$ and $\eta>0$. Let $0<\mu<\frac{2 \eta}{\kappa^{2}}$ and $0<t<$ $\sigma \leq 1$. Then $\sigma I-t \mu G: H \rightarrow H$ is a contractive mapping with a constant $\sigma-t \tau$, where $\tau=$ $1-\sqrt{1-\mu\left(2 \eta-\mu \kappa^{2}\right)}$.

Lemma 2.9. [10] Assume that $T$ is nonexpansive self mapping of a closed convex subset of $C$ of a Hilbert space $H$. If $T$ has a fixed point, then $I-T$ is demiclosed, i.e., whenever $\left\{x_{n}\right\}$ is a sequence in $C$ converging weakly to some $x \in C$ and the sequence $\left\{(I-T) x_{n}\right\}$ converges strongly to some $y$, it follows that $(I-T) x=y$, where I is the identity mapping $H$.

In the following, we write $x_{n} \rightarrow x$ to indicate that the sequence $\left\{x_{n}\right\}$ converges weakly to $x$. $x_{n} \rightarrow x$ implies that $\left\{x_{n}\right\}$ converges strongly to $x$.

\section{ITERATIVE ALGORITHMS}

Throughout the rest of this paper, we always assume the following:

- $H_{1}$ and $H_{2}$ are real Hilbert spaces with the inner product $\langle\cdot, \cdot\rangle$ and the induced norm $\|\cdot\|$;

- $A: H_{1} \rightarrow H_{2}$ is a bounded linear operator;

- $A^{*}: H_{2} \rightarrow H_{1}$ is the adjoint of $A$;

- $L$ is the spectral radius of the operator $A^{*} A$

- $B: H_{1} \rightarrow 2^{H_{1}}$ is a maximal monotone operator with $\operatorname{dom}(B) \subset H_{1}$;

- $B^{-1} 0$ is the set of zero points of $B$, that is, $B^{-1} 0=\left\{z \in H_{1}: 0 \in B z\right\}$;

- $J_{\lambda_{n}}^{B}: H_{1} \rightarrow \operatorname{dom}(B)$ is the resolvent of $B$ for $\lambda_{n} \in(0, \infty)$ and $\liminf _{n \rightarrow \infty} \lambda_{n}>0$;

- $G: H_{1} \rightarrow H_{1}$ is a $\kappa$-Lipschitzian and $\eta$-strongly monotone mapping with constants $\kappa, \eta>0$

- $V: H_{1} \rightarrow H_{1}$ is an $l$-Lipschitzian mapping with constant $l \in[0, \infty)$;

- Constants $\mu>0$ and $\gamma \geq 0$ satisfy $0<\mu<\frac{2 \eta}{\kappa^{2}}$ and $0 \leq \gamma l<\tau$, where $\tau=1-\sqrt{1-\mu\left(2 \eta-\mu \kappa^{2}\right)}$;

- $T: H_{1} \rightarrow H_{1}$ is a continuous pseudocontractive mapping with $\operatorname{Fix}(T) \neq \emptyset$;

- $T_{r_{n}}: H_{1} \rightarrow H_{1}$ is a mapping defined by

$$
T_{r_{n}} x=\left\{z \in H_{1}:\langle T z, y-z\rangle-\frac{1}{r_{n}}\left\langle y-z,\left(1+r_{n}\right) z-x\right\rangle \leq 0, \quad \forall y \in H_{1}\right\}
$$

for $x \in H_{1}$ and $r_{n} \in(0, \infty)$, and $\liminf _{n \rightarrow \infty} r_{n}>0$;

- $R: H_{2} \rightarrow H_{2}$ is a continuous pseudocontractive mapping with $\operatorname{Fix}(R) \neq \emptyset$;

- $R_{v_{n}}: H_{2} \rightarrow H_{2}$ is a mapping defined by

$$
R_{v_{n}} x=\left\{z \in H_{2}:\langle R z, y-z\rangle-\frac{1}{v_{n}}\left\langle y-z,\left(1+v_{n}\right) z-x\right\rangle \leq 0, \quad \forall y \in H_{2}\right\}
$$

for $x \in H$ and $v_{n} \in(0, \infty)$, and $\liminf _{n \rightarrow \infty} v_{n}>0$; 
- $\Omega:=B^{-1} 0 \cap A^{-1}(F i x(R)) \cap F i x(T) \neq \emptyset$.

By Lemma 2.6, we note that $T_{r_{n}}$ and $R_{V_{n}}$ are firmly nonexpansive, and $F i x\left(T_{r_{n}}\right)=F i x(T)$ and $\operatorname{Fix}\left(R_{v_{n}}\right)=\operatorname{Fix}(R)$.

Now, we propose a new general iterative algorithm, which generates a sequence $\left\{x_{n}\right\}$ in an explicit way: for an arbitrarily chosen $x_{0} \in C$,

$$
\left\{\begin{aligned}
z_{n} & =J_{\lambda_{n}}^{B}\left(x_{n}+\eta_{n} A^{*}\left(R_{v_{n}}-I\right) A x_{n}\right), \\
x_{n+1} & =\beta_{n} x_{n}+\left(1-\beta_{n}\right) T_{r_{n}}\left(\alpha_{n} \gamma V x_{n}+\left(I-\alpha_{n} \mu G\right) z_{n}\right), \quad n \geq 0,
\end{aligned}\right.
$$

where $\left\{\alpha_{n}\right\}$ and $\left\{\beta_{n}\right\}$ are two sequences in $(0,1)$, and $\left\{r_{n}\right\},\left\{\lambda_{n}\right\},\left\{v_{n}\right\} \subset(0, \infty)$, and $\left\{\eta_{n}\right\} \subset$ $\left(0, \frac{1}{L}\right)$ and establish strong convergence of this sequence to an element in $\Omega$.

Theorem 3.1. Let the sequence $\left\{x_{n}\right\}$ be generated iteratively by explicit algorithm (3.1). Let $\left\{\alpha_{n}\right\},\left\{\beta_{n}\right\} \subset(0,1),\left\{r_{n}\right\},\left\{\lambda_{n}\right\},\left\{v_{n}\right\} \subset(0, \infty)$ and $\left\{\eta_{n}\right\} \subset\left(0, \frac{1}{L}\right)$ satisfy the following conditions:

(C1) $\lim _{n \rightarrow \infty} \alpha_{n}=0$;

(C2) $\sum_{n=1}^{\infty} \alpha_{n}=\infty$

(C3) $0<\liminf _{n \rightarrow \infty} \beta_{n} \leq \limsup _{n \rightarrow \infty} \beta_{n}<1$;

(C4) $0<r \leq r_{n}<\infty$ and $\lim _{n \rightarrow \infty}\left|r_{n+1}-r_{n}\right|=0$;

(C5) $0<\lambda \leq \lambda_{n}<\infty$ and $\lim _{n \rightarrow \infty}\left|\lambda_{n+1}-\lambda_{n}\right|=0$;

(C6) $0<v \leq v_{n}<\infty$ and $\lim _{n \rightarrow \infty}\left|v_{n+1}-\mu_{n}\right|=0$;

(C7) $0<\eta \leq \eta_{n}<\frac{1}{L}$ and $\lim _{n \rightarrow \infty}\left|\eta_{n+1}-\eta_{n}\right|=0$.

Then $\left\{x_{n}\right\}$ converges strongly to a point $q \in \Omega$, which is the unique solution of the variational inequality

$$
\langle(\mu G-\gamma V) q, p-q\rangle \geq 0, \quad \forall p \in \Omega
$$

Proof. First, let $Q=P_{\Omega}$. Then, by the closedness and convexity of $\Omega$ (due to Lemma 2.2 and Lemma 2.6 (iv)), $P_{\Omega}$ is well-defined. Also, it is easy to show that $Q(I-\mu G+\gamma V): H_{1} \rightarrow H_{1}$ is a contractive mapping with the constant $1-(\tau-\gamma l)$. In fact, from Lemma 2.8, we have

$$
\begin{aligned}
\|Q(I-\mu G+\gamma V) x-Q(I-\mu G+\gamma V) y\| \\
\quad \leq\|(I-\mu G+\gamma V) x-(I-\mu G+\gamma V) y\| \\
\quad \leq\|(I-\mu G) x-(I-\mu G) y\|+\gamma\|V x-V y\| \\
\quad \leq(1-(\tau-\gamma l))\|x-y\|
\end{aligned}
$$

for any $x, y \in H_{1}$. So, $Q(I-\mu G+\gamma V)$ is a contractive mapping with the constant $1-(\tau-$ $\gamma l)<1$. Thus, by Banach contraction principle, there exists a unique element $q \in H_{1}$ such that $q=P_{\Omega}(I-\mu G+\gamma V) q$. Equivalently, $q$ is a solution of the variational inequality (3.2) by (2.5). We note that the uniqueness of a solution of the variational inequality (3.2) is a consequence of the strong monotonicity of $\mu G-\gamma V$ due to Lemma 2.7 (see $[12,13]$ for this fact). Below we will use $q \in \Omega$ to denote the unique solution of variational inequality (3.2). 
From now on, we put $u_{n}=x_{n}+\eta_{n} A^{*}\left(R_{v_{n}}-I\right) A x_{n}=\left(I+\eta_{n} A^{*}\left(R_{v_{n}}-I\right) A\right) x_{n}=K_{n} x_{n}, y_{n}=$ $\alpha_{n} \gamma V x_{n}+\left(I-\alpha_{n} \mu G\right) J_{\lambda_{n}}^{B} u_{n}$ and $z_{n}=J_{\lambda_{n}}^{B} u_{n}$ for $n \geq 0$. Let $p \in \Omega$. Since

$$
\begin{aligned}
\left\|z_{n}-p\right\|^{2} & =\left\|J_{\lambda_{n} t}^{B} u_{n}-J_{\lambda_{n}}^{B} p\right\|^{2} \\
& =\| J_{\lambda_{n}}^{B}\left(x_{n}+\eta_{t} A^{*}\left(R_{v_{n}}-I\right) A x_{n}-J_{\lambda_{n}}^{B} p \|^{2}\right. \\
& \leq\left\|x_{n}+\eta_{n} A^{*}\left(R_{v_{n}}-I\right) A x_{n}-p\right\|^{2} \\
& =\left\|x_{n}-p\right\|^{2}+\eta_{n}^{2}\left\|A^{*}\left(R_{v_{n}}-I\right) A x_{n}\right\|^{2}+2 \eta_{n}\left\langle x_{n}-p, A^{*}\left(R_{v_{n}}-I\right) A x_{n}\right\rangle,
\end{aligned}
$$

we have

$$
\begin{aligned}
\left\|z_{n}-p\right\|^{2}= & \left\|J_{\lambda_{n}}^{B} u_{n}-J_{\lambda_{n}}^{B} p\right\|^{2} \\
\leq & \left.\left\|x_{n}-p\right\|^{2}+\eta_{n}^{2}\left\langle R_{v_{n}}-I\right) A x_{n}, A A^{*}\left(R_{v_{n}}-I\right) A x_{n}\right\rangle \\
& +2 \eta_{n}\left\langle x_{n}-p, A^{*}\left(R_{v_{n}}-I\right) A x_{n}\right\rangle .
\end{aligned}
$$

And, we see that

$$
\begin{aligned}
\left.\eta_{n}^{2}\left\langle R_{v_{n}}-I\right) A x_{n}, A A^{*}\left(R_{v_{n}}-I\right) A x_{n}\right\rangle & \leq L \eta_{n}^{2}\left\langle\left(R_{v_{n}}-I\right) A x_{n},\left(R_{v_{n}}-I\right) A x_{n}\right\rangle \\
& =L \eta_{n}^{2}\left\|\left(R_{v_{n}}-I\right) A x_{n}\right\|^{2} .
\end{aligned}
$$

Moreover, from (2.6), we obtain

$$
\begin{aligned}
2 \eta_{n}\left\langle x_{n}-\right. & \left.p, A^{*}\left(R_{v_{n}}-I\right) A x_{n}\right\rangle \\
& =2 \eta_{n}\left\langle A\left(x_{n}-p\right),\left(R_{v_{n}}-I\right) A x_{n}\right\rangle \\
& =2 \eta_{n}\left\langle A\left(x_{n}-p\right)+\left(R_{v_{n}}-I\right) A x_{n}-\left(R_{v_{n}}-I\right) A x_{n},\left(R_{v_{n}}-I\right) A x_{n}\right\rangle \\
& =2 \eta_{n}\left[\left\langle\left(R_{v_{n}}\left(A x_{n}\right)-A p,\left(R_{v_{n}}-I\right) A x_{n}\right\rangle-\left\|\left(R_{v_{n}}-I\right) A x_{n}\right\|^{2}\right]\right. \\
& \leq 2 \eta_{n}\left[\frac{1}{2}\left\|\left(R_{v_{n}}-I\right) A x_{n}\right\|^{2}-\left\|\left(R_{v_{n}}-I\right) A x_{n}\right\|^{2}\right] \\
& =-\eta_{n}\left\|\left(R_{v_{n}}-I\right) A x_{n}\right\|^{2} .
\end{aligned}
$$

Therefore, from (3.3), (3.4), (3.5) and (3.6), we derive

$$
\begin{aligned}
\left\|z_{n}-p\right\|^{2} & =\left\|J_{\lambda_{n}}^{B} u_{n}-J_{\lambda_{n}}^{B} p\right\|^{2} \\
& \leq\left\|u_{n}-p\right\|^{2} \\
& =\left\|x_{n}+\eta_{n} A^{*}\left(R_{v_{n}}-I\right) A x_{n}-p\right\|^{2} \\
& \leq\left\|x_{n}-p\right\|^{2}+\eta_{n}\left(L \eta_{n}-1\right)\left\|\left(R_{v_{t}}-I\right) A x_{n}\right\|^{2} \\
& \leq\left\|x_{n}-p\right\|^{2} \quad\left(\text { by } \eta_{n} \in\left(0, \frac{1}{L}\right)\right) .
\end{aligned}
$$

Now, we divide the proof into several steps. 
Step 1. We show that $\left\{x_{n}\right\}$ is bounded. First, noting that $p=J_{\lambda_{n}}^{B} p, p=K_{n} p$ and $p=T_{r_{n}} p$, we obtain

$$
\begin{aligned}
\left\|y_{n}-p\right\|= & \left\|\alpha_{n} \gamma V x_{n}+\left(I-\alpha_{n} \mu G\right) J_{r_{n}}^{B} u_{n}-p\right\| \\
\leq & \left\|\alpha_{n}\left(\gamma V x_{n}-\gamma V p\right)+\alpha_{n}(\gamma V p-\mu G p)\right\| \\
& +\left\|\left(I-\alpha_{n} \mu G\right) J_{\lambda_{n}}^{B} u_{n}-\left(I-\alpha_{n} \mu G\right) p\right\| \\
\leq & \alpha_{n}\left\|\gamma V x_{n}-\gamma V p\right\|+\alpha_{n}\|\gamma V p-\mu G p\|+\left(1-\alpha_{n} \tau\right)\left\|u_{n}-p\right\| \\
\leq & \alpha_{n} \gamma l\left\|x_{n}-p\right\|+\alpha_{n}\|\gamma V p-\mu G p\|+\left(1-\alpha_{n} \tau\right)\left\|x_{n}-p\right\| \\
= & \left(1-(\tau-\gamma l) \alpha_{n}\right)\left\|x_{n}-p\right\|+\alpha_{n}\|\gamma V p-\mu G p\| .
\end{aligned}
$$

Thus, since $T_{r_{n}}$ is nonexpansive (by Lemma 2.6), from (3.1) and (3.8), we deduce

$$
\begin{aligned}
\left\|x_{n+1}-p\right\| & \leq \beta_{n}\left\|x_{n}-p\right\|+\left(1-\beta_{n}\right)\left\|T_{r_{n}} y_{n}-p\right\| \\
& \leq \beta_{n}\left\|x_{n}-p\right\|+\left(1-\beta_{n}\right)\left\|y_{n}-p\right\| \\
& \leq \beta_{n}\left\|x_{n}-p\right\|+\left(1-\beta_{n}\right)\left[\left(1-(\tau-\gamma l) \alpha_{n}\right)\left\|x_{n}-p\right\|+\alpha_{n}\|\gamma V p-\mu G p\|\right] \\
& =\left(1-\left(1-\beta_{n}\right)(\tau-\gamma l) \alpha_{n}\right)\left\|x_{n}-p\right\|+\left(1-\beta_{n}\right)(\tau-\gamma l) \alpha_{n} \frac{\|\gamma V p-\mu G p\|}{\tau-\gamma l} \\
& \leq \max \left\{\left\|x_{n}-p\right\|, \frac{\|\gamma V p-\mu G p\|}{\tau-\gamma l}\right\} .
\end{aligned}
$$

Using an induction, we have

$$
\left\|x_{n}-p\right\| \leq \max \left\{\frac{\|\gamma V p-\mu G p\|}{\tau-\gamma l},\left\|x_{0}-p\right\|\right\} .
$$

Hence, $\left\{x_{n}\right\}$ is bounded. Also, $\left\{y_{n}\right\},\left\{u_{n}\right\}\left(=\left\{K_{n} x_{n}\right\}\right) z_{n}=\left\{J_{\lambda_{n}}^{B} u_{n}\right\},\left\{G x_{n}\right\},\left\{G J_{\lambda_{n}}^{B} u_{n}\right\},\left\{w_{n}\right\}$ $=\left\{T_{r_{n}} y_{n}\right\}$ and $\left\{V x_{n}\right\}$ are bounded. And, from condition (C1), it follows that

$$
\left\|y_{n}-z_{n}\right\|=\left\|y_{n}-J_{\lambda_{n}}^{B} u_{n}\right\|=\alpha_{n}\left\|\gamma V x_{n}-\mu G J_{\lambda_{n}}^{B} u_{n}\right\| \rightarrow 0 \text { as } n \rightarrow \infty .
$$

Step 2. We show that $\lim _{n \rightarrow \infty}\left\|x_{n+1}-x_{n}\right\|=0$. For this purpose, first, we derive

$$
\begin{aligned}
\left\|y_{n}-y_{n-1}\right\|= & \| \alpha_{n} \gamma V x_{n}+\left(I-\alpha_{n} \mu G\right) J_{\lambda_{n}}^{B} u_{n} \\
& \quad-\left(\alpha_{n-1} \gamma V x_{n-1}+\left(I-\alpha_{n-1} \mu G\right) J_{\lambda_{n-1}}^{B} u_{n-1}\right) \| \\
\leq & \left\|\left(\alpha_{n}-\alpha_{n-1}\right) \gamma V x_{n-1}+\alpha_{n}\left(\gamma V x_{n}-\gamma V x_{n-1}\right)\right\| \\
& +\left\|\left(I-\alpha_{n} \mu G\right) J_{\lambda_{n}}^{B} u_{n}-\left(I-\alpha_{n} \mu G\right) J_{\lambda_{n-1}}^{B} u_{n-1}\right\| \\
& +\left\|\left(I-\alpha_{n} \mu G\right) J_{\lambda_{n-1}}^{B} u_{n-1}-\left(I-\alpha_{n-1} \mu G\right) J_{\lambda_{n-1}}^{B} u_{n-1}\right\| \\
\leq & \left|\alpha_{n}-\alpha_{n-1}\right|\left\|\gamma V x_{n-1}\right\|+\alpha_{n} \gamma l\left\|x_{n}-x_{n-1}\right\| \\
& +\left(1-\alpha_{n} \tau\right)\left\|J_{\lambda_{n}}^{B} u_{n}-J_{\lambda_{n-1}}^{B} u_{n-1}\right\|+\left|\alpha_{n}-\alpha_{n+1}\right|\left\|\mu G J_{\lambda_{n-1}}^{B} u_{n-1}\right\| \\
= & \left|\alpha_{n}-\alpha_{n-1}\right|\left(\left\|\gamma V x_{n-1}\right\|+\left\|\mu G J_{\lambda_{n-1}}^{B} u_{n-1}\right\|\right) \\
& +\alpha_{n} \gamma l\left\|x_{n}-x_{n-1}\right\|+\left(1-\alpha_{n} \tau\right)\left\|J_{\lambda_{n}}^{B} u_{n}-J_{\lambda_{n-1}}^{B} u_{n-1}\right\| \\
\leq & \left|\alpha_{n}-\alpha_{n-1}\right| M_{1}+\alpha_{n} \gamma l\left\|x_{n}-x_{n-1}\right\|+\left(1-\alpha_{n} \tau\right)\left\|J_{\lambda_{n}}^{B} u_{n}-J_{\lambda_{n-1}}^{B} u_{n-1}\right\|,
\end{aligned}
$$


where $M_{1}>0$ is an appropriate constant. And, from the Resolvent Identity (2.2) and condition (C5) $\left(0<\lambda \leq \lambda_{n}\right.$ for $\left.n \geq 0\right)$, we induce

$$
\begin{aligned}
\left\|J_{\lambda_{n}}^{B} u_{n}-J_{\lambda_{n-1}}^{B} u_{n-1}\right\| & =\left\|J_{\lambda_{n-1}}^{B}\left(\frac{\lambda_{n-1}}{\lambda_{n}} u_{n}+\left(1-\frac{\lambda_{n-1}}{\lambda_{n}}\right) J_{\lambda_{n}}^{B} u_{n}\right)-J_{\lambda_{n-1}}^{B} u_{n-1}\right\| \\
& \leq\left\|\frac{\lambda_{n-1}}{\lambda_{n}}\left(u_{n}-u_{n-1}\right)+\left(1-\frac{\lambda_{n-1}}{\lambda_{n}}\right)\left(J_{\lambda_{n}}^{B} u_{n}-u_{n-1}\right)\right\| \\
& \leq\left\|u_{n}-u_{n-1}\right\|+\frac{\left|\lambda_{n}-\lambda_{n-1}\right|}{\lambda_{n}}\left\|J_{\lambda_{n}}^{B} u_{n}-u_{n}\right\| \\
& \leq\left\|u_{n}-u_{n-1}\right\|+\frac{\left|\lambda_{n}-\lambda_{n-1}\right|}{\lambda} M_{2},
\end{aligned}
$$

where $M_{2}>0$ is an appropriate constant. Again, since $K_{n}=I+\eta_{n} A^{*}\left(R_{v_{n}}-I\right) A$ is nonexpansive as averaged (Lemma 2.1), we calculate

$$
\begin{aligned}
\left\|u_{n}-u_{n-1}\right\|= & \left\|\left(I+\eta_{n} A^{*}\left(R_{v_{n}}-I\right) A\right) x_{n}-\left(I+\eta_{n-1} A^{*}\left(R_{v_{n-1}}-I\right) A\right) x_{n-1}\right\| \\
= & \left\|K_{n} x_{n}-K_{n-1} x_{n-1}\right\| \\
\leq & \left\|K_{n} x_{n}-K_{n} x_{n-1}\right\|+\left\|K_{n} x_{n-1}-K_{n-1} x_{n-1}\right\| \\
\leq & \left\|x_{n}-x_{n-1}\right\| \\
& \quad+\left\|\left(x_{n-1}+\eta_{n} A^{*}\left(R_{v_{n}}-I\right) A x_{n-1}\right)-\left(x_{n-1}+\eta_{n-1} A^{*}\left(R_{v_{n-1}}-I\right) A x_{n-1}\right)\right\| \\
\leq & \left\|x_{n}-x_{n-1}\right\|+\left\|\eta_{n} A^{*}\left(R_{v_{n}}-I\right) A x_{n-1}-\eta_{n-1} A^{*}\left(R_{v_{n}}-I\right) A x_{n-1}\right\| \\
& \quad+\left\|\eta_{n-1} A^{*}\left(R_{v_{n}}-I\right) A x_{n-1}-\eta_{n-1} A^{*}\left(R_{v_{n-1}}-I\right) A x_{n-1}\right\| \\
\leq & \left\|x_{n}-x_{n-1}\right\|+\left|\eta_{n}-\eta_{n-1}\right|\left\|A^{*}\left(R_{v_{n}}-I\right) A x_{n-1}\right\| \\
& \quad+\eta_{n-1}\left\|A^{*}\right\|\left\|R_{v_{n}}\left(A x_{n-1}\right)-R_{v_{n-1}}\left(A x_{n-1}\right)\right\| \\
\leq & \left\|x_{n}-x_{n-1}\right\|+\left|\eta_{n}-\eta_{n-1}\right| M_{3}+\frac{1}{L}\left\|A^{*}\right\|\left\|R_{v_{n}}\left(A x_{n-1}\right)-R_{v_{n-1}}\left(A x_{n-1}\right)\right\|,
\end{aligned}
$$

where $M_{3}>0$ is an appropriate constant. Let $R_{v_{n}}\left(A x_{n-1}\right)=d_{n}^{\prime}$ and $R_{v_{n-1}}\left(A x_{n-1}\right)=d_{n-1}$. Then, by Lemma 2.6, we have

$$
\left\langle y-d_{n}^{\prime}, R d_{n}^{\prime}\right\rangle-\frac{1}{v_{n}}\left\langle y-d_{n}^{\prime},\left(1+v_{n}\right) d_{n}^{\prime}-A x_{n-1}\right\rangle \leq 0, \quad \forall y \in H_{2}
$$

and

$$
\left\langle y-d_{n-1}, R d_{n-1}\right\rangle-\frac{1}{v_{n-1}}\left\langle y-d_{n-1},\left(1+v_{n-1}\right) d_{n-1}-A x_{n-1}\right\rangle \leq 0, \quad \forall y \in H_{2} .
$$

Putting $y:=d_{n-1}$ in (3.13) and $y:=d_{n}^{\prime}$ in (3.14), we get

$$
\left\langle d_{n-1}-d_{n}^{\prime}, R d_{n}^{\prime}\right\rangle-\frac{1}{v_{n}}\left\langle d_{n-1}-d_{n}^{\prime},\left(1+v_{n}\right) d_{n}^{\prime}-A x_{n-1}\right\rangle \leq 0
$$

and

$$
\left\langle d_{n}^{\prime}-d_{n-1}, R d_{n-1}\right\rangle-\frac{1}{v_{n-1}}\left\langle d_{n}^{\prime}-d_{n-1},\left(1+v_{n-1}\right) d_{n-1}-A x_{n-1}\right\rangle \leq 0 .
$$


Adding up (3.15) and (3.16), we obtain

$$
\begin{aligned}
& \left\langle d_{n-1}-d_{n}^{\prime}, R d_{n}^{\prime}-R d_{n-1}\right\rangle \\
& -\left\langle d_{n-1}-d_{n}^{\prime}, \frac{\left(1+v_{n}\right) d_{n}^{\prime}-A x_{n-1}}{v_{n}}-\frac{\left(1+v_{n-1}\right) d_{n-1}-A x_{n-1}}{v_{n-1}}\right\rangle \leq 0 .
\end{aligned}
$$

Since $R$ is pseudocontractive, by (3.17), we have

$$
\left\langle d_{n-1}-d_{n}^{\prime}, \frac{d_{n}^{\prime}-A x_{n-1}}{v_{n}}-\frac{d_{n-1}-A x_{n-1}}{v_{n-1}}\right\rangle \geq 0,
$$

and hence

$$
\left\langle d_{n-1}-d_{n}^{\prime}, d_{n}^{\prime}-d_{n-1}+d_{n-1}-A x_{n-1}-\frac{v_{n}}{v_{n-1}}\left(d_{n-1}-A x_{n-1}\right)\right\rangle \geq 0 .
$$

From (3.18) and condition (C6) $\left(0<v \leq v_{n}\right.$ for $\left.n \geq 0\right)$, we derive

$$
\begin{aligned}
\left\|d_{n}^{\prime}-d_{n-1}\right\|^{2} & \leq\left\langle d_{n-1}-d_{n}^{\prime},\left(1-\frac{v_{n}}{v_{n-1}}\right)\left(d_{n-1}-A x_{n-1}\right)\right\rangle \\
& \leq\left\|d_{n-1}-d_{n}^{\prime}\right\|\left|v_{n}-v_{n-1}\right| \frac{\left\|d_{n-1}-A x_{n-1}\right\|}{v_{n-1}}
\end{aligned}
$$

and hence

$$
\left\|R_{v_{n}}\left(A x_{n-1}\right)-R_{v_{n-1}}\left(A x_{n-1}\right)\right\|=\left\|d_{n}^{\prime}-d_{n-1}\right\| \leq\left|v_{n}-v_{n-1}\right| \frac{M_{4}}{v},
$$

where $M_{4}>0$ is an appropriate constant.

Now, substituting (3.19) into (3.12), we get

$$
\left\|u_{n}-u_{n-1}\right\| \leq\left\|x_{n}-x_{n-1}\right\|+\left|\eta_{n}-\eta_{n-1}\right| M_{3}+\frac{1}{L}\left\|A^{*}\right\|\left|v_{n}-v_{n-1}\right| \frac{M_{4}}{v} .
$$

From (3.10), (3.11) and (3.20), we drive

$$
\begin{aligned}
\left\|y_{n}-y_{n-1}\right\| \leq & \left|\alpha_{n}-\alpha_{n-1}\right| M_{1}+\alpha_{n} \gamma l\left\|x_{n}-x_{n-1}\right\| \\
& +\left(1-\alpha_{n} \tau\right)\left\|J_{\lambda_{n}}^{B} u_{n}-J_{\lambda_{n-1}}^{B} u_{n-1}\right\| \\
\leq & \left|\alpha_{n}-\alpha_{n-1}\right| M_{1}+\alpha_{n} \gamma l\left\|x_{n}-x_{n-1}\right\| \\
& +\left(1-\alpha_{n} \tau\right)\left(\left\|u_{n}-u_{n-1}\right\|+\left|\lambda_{n}-\lambda_{n-1}\right| \frac{M_{2}}{\lambda}\right) \\
\leq & \left|\alpha_{n}-\alpha_{n-1}\right| M_{1}+\alpha_{n} \gamma l\left\|x_{n}-x_{n-1}\right\| \\
& +\left(1-\alpha_{n} \tau\right)\left[\left\|x_{n}-x_{n-1}\right\|+\left|\eta_{n}-\eta_{n-1}\right| M_{3}\right. \\
& \left.+\frac{1}{L}\left\|A^{*}\right\|\left|v_{n}-v_{n-1}\right| \frac{M_{4}}{v}+\left|\lambda_{n}-\lambda_{n-1}\right| \frac{M_{2}}{\lambda}\right] .
\end{aligned}
$$

On the other hand, let $w_{n}=T_{r_{n}} y_{n}$. Since $w_{r_{n-1}}=T_{r_{n-1}} y_{n-1}$, we have

$$
\left\langle y-w_{n}, T w_{n}\right\rangle-\frac{1}{r_{n}}\left\langle y-w_{n},\left(1+r_{n}\right) w_{n}-y_{n}\right\rangle \leq 0, \quad \forall y \in H_{1},
$$

and

$$
\left\langle y-w_{n-1}, T w_{n-1}\right\rangle-\frac{1}{r_{n-1}}\left\langle y-w_{n-1},\left(1+r_{n-1}\right) w_{n-1}-y_{n-1}\right\rangle \leq 0, \quad \forall y \in H_{1} .
$$


Putting $y:=z_{n-1}$ in (3.22) and $y:=z_{n}$ in (3.23), we get

$$
\left\langle w_{n-1}-w_{n}, T w_{n}\right\rangle-\frac{1}{r_{n}}\left\langle w_{n-1}-w_{n},\left(1+r_{n}\right) w_{n}-y_{n}\right\rangle \leq 0
$$

and

$$
\left\langle w_{n}-w_{n-1}, T w_{n-1}\right\rangle-\frac{1}{r_{n-1}}\left\langle w_{n}-w_{n-1},\left(1+r_{n-1}\right) w_{n-1}-y_{n-1}\right\rangle \leq 0 .
$$

Adding up (3.24) and (3.25), we obtain

$$
\begin{aligned}
\left\langle w_{n-1}-\right. & \left.w_{n}, T w_{n}-T w_{n-1}\right\rangle \\
& -\left\langle w_{n-1}-w_{n}, \frac{\left(1+r_{n}\right) w_{n}-y_{n}}{r_{n}}-\frac{\left(1+r_{n-1}\right) w_{n-1}-y_{n-1}}{r_{n-1}}\right\rangle \leq 0 .
\end{aligned}
$$

Using the fact that $T$ is pseudocontractive, we have by (3.26) that

$$
\left\langle w_{n-1}-w_{n}, \frac{w_{n}-y_{n}}{r_{n}}-\frac{w_{n-1}-y_{n-1}}{r_{n-1}}\right\rangle \geq 0,
$$

and hence

$$
\left\langle w_{n-1}-w_{n}, w_{n}-w_{n-1}+w_{n-1}-y_{n}-\frac{r_{n}}{r_{n-1}}\left(w_{n-1}-y_{n-1}\right)\right\rangle \geq 0 .
$$

From (3.27) and condition (C4) $\left(0<r \leq r_{n}\right.$ for $\left.n \geq 0\right)$, we derive

$$
\begin{aligned}
\left\|w_{n}-w_{n-1}\right\|^{2} & \leq\left\langle w_{n-1}-w_{n}, y_{n-1}-y_{n}+\left(1-\frac{r_{n}}{r_{n-1}}\right)\left(w_{n-1}-y_{n-1}\right)\right\rangle \\
& \leq\left\|w_{n-1}-w_{n}\right\|\left(\left\|y_{n-1}-y_{n}\right\|+\frac{\left|r_{n}-r_{n-1}\right|}{r}\left\|w_{n-1}-y_{n-1}\right\|\right) .
\end{aligned}
$$

Thus we obtain

$$
\left\|w_{n}-w_{n-1}\right\| \leq\left\|y_{n-1}-y_{n}\right\|+\frac{\left|r_{n}-r_{n-1}\right|}{r} M_{5},
$$

where $M_{5}$ is an appropriate constant. Substituting (3.21) into (3.28) yields

$$
\begin{aligned}
\left\|w_{n}-w_{n-1}\right\|= & \left\|T_{r_{n}} y_{n}-T_{r_{n-1}} y_{n-1}\right\| \\
\leq & \left(1-\alpha_{n}(\tau-\gamma l)\right)\left\|x_{n}-x_{n-1}\right\|+\left|\alpha_{n}-\alpha_{n-1}\right| M_{1}+\left|\eta_{n}-\eta_{n-1}\right| M_{3} \\
& +\frac{1}{L} \| A^{*}||\left|v_{n}-v_{n-1}\right| \frac{M_{4}}{v}+\left|\lambda_{n}-\lambda_{n-1}\right| \frac{M_{2}}{\lambda}+\left|r_{n}-r_{n-1}\right| \frac{M_{5}}{r} .
\end{aligned}
$$

In view of conditions (C1), (C4), (C5), (C6) and (C7), we find from (3.29) that

$$
\underset{n \rightarrow \infty}{\limsup }\left(\left\|w_{n}-w_{n-1}\right\|-\left\|x_{n}-x_{n-1}\right\| \leq 0 .\right.
$$

Thus, by Lemma 2.4, we have

$$
\lim _{n \rightarrow \infty}\left\|w_{n}-x_{n}\right\|=\lim _{n \rightarrow \infty}\left\|T_{r_{n}} y_{n}-x_{n}\right\|=0 .
$$

Since $x_{n+1}-x_{n}=\left(1-\beta_{n}\right)\left(z_{n}-x_{n}\right)$, by (3.30) and condition (C3), we conclude

$$
\lim _{n \rightarrow \infty}\left\|x_{n+1}-x_{n}\right\|=0 .
$$


Step 3. We show that $\lim _{n \rightarrow \infty}\left\|u_{n}-z_{n}\right\|=\lim _{n \rightarrow \infty}\left\|u_{n}-J_{\lambda_{n}}^{B} u_{n}\right\|=0$. To this end, first, by (3.7), we see that

$$
\begin{aligned}
\left\|u_{n}-p\right\|^{2} & =\left\|x_{n}+\eta_{n} A^{*}\left(R_{v_{n}}-I\right) A x_{n}-p\right\|^{2} \\
& \leq\left\|x_{n}-p\right\|^{2}+\eta_{n}\left(L \eta_{n}-1\right)\left\|\left(R_{v_{t}}-I\right) A x_{n}\right\|^{2} \\
& \leq\left\|x_{n}-p\right\|^{2} \quad\left(\text { by } \eta_{n} \in\left(0, \frac{1}{L}\right)\right) .
\end{aligned}
$$

Again, since $J_{\lambda_{n}}^{B}$ is firmly nonexpansive ((2.1)), we have

$$
\begin{aligned}
\left\|z_{n}-p\right\|^{2} & \leq\left\langle J_{\lambda_{n}}^{B} u_{n}-J_{\lambda_{n}}^{B} p, u_{n}-p\right\rangle \\
& =\frac{1}{2}\left[\left\|u_{n}-p\right\|^{2}+\left\|z_{n}-p\right\|^{2}-\left\|u_{n}-z_{n}\right\|^{2}\right] .
\end{aligned}
$$

Hence

$$
\begin{aligned}
\left\|z_{n}-p\right\|^{2} & \leq\left\|u_{n}-p\right\|^{2}-\left\|u_{n}-z_{n}\right\|^{2} \\
& \leq\left\|x_{n}-p\right\|^{2}-\left\|u_{n}-z_{n}\right\|^{2} .
\end{aligned}
$$

Thus, by (2.4), (3.7) and (3.32), we obtain

$$
\begin{aligned}
\left\|x_{n+1}-p\right\|^{2} \leq & \beta_{n}\left\|x_{n}-p\right\|^{2}+\left(1-\beta_{n}\right)\left\|T_{r_{n}} y_{n}-p\right\|^{2} \\
\leq & \beta_{n}\left\|x_{n}-p\right\|^{2}+\left(1-\beta_{n}\right)\left\|y_{n}-p\right\|^{2} \\
= & \beta_{n}\left\|x_{n}-p\right\|^{2} \\
& +\left(1-\beta_{n}\right)\left\|\alpha_{n}\left(\gamma V x_{n}-\mu G p\right)+\left(I-\alpha_{n} \mu G\right) J_{\lambda_{n}}^{B} u_{n}-\left(I-\alpha_{n} \mu G\right) p\right\|^{2} \\
\leq & \beta_{n}\left\|x_{n}-p\right\|^{2}+\left(1-\beta_{n}\right)\left(\alpha_{n}\left\|\gamma V x_{n}-\mu G p\right\|+\left(1-\alpha_{n} \tau\right)\left\|z_{n}-p\right\|\right)^{2} \\
\leq & \beta_{n}\left\|x_{n}-p\right\|^{2}+\left(1-\beta_{n}\right)\left(\alpha_{n}\left\|\gamma V x_{n}-\mu G p\right\|+\left\|z_{n}-p\right\|\right)^{2} \\
\leq & \beta_{n}\left\|x_{n}-p\right\|^{2}+\left(1-\beta_{n}\right)\left[\alpha_{n} M_{6}+\left\|x_{n}-p\right\|^{2}-\left\|u_{n}-z_{n}\right\|^{2}\right] \\
\leq & \left\|x_{n}-p\right\|^{2}+\left(1-\beta_{n}\right)\left(\alpha_{n} M_{6}-\left\|u_{n}-z_{n}\right\|^{2}\right),
\end{aligned}
$$

where $M_{6}>0$ is an appropriate constant. So,

$$
\begin{aligned}
\left\|u_{n}-z_{n}\right\|^{2} & \leq \alpha_{n} M_{6}+\frac{1}{1-\beta_{n}}\left(\left\|x_{n}-p\right\|^{2}-\left\|x_{n+1}-p\right\|^{2}\right) \\
& \leq \alpha_{n} M_{6}+\frac{1}{1-\beta_{n}}\left(\left\|x_{n}-p\right\|+\left\|x_{n-1}-p\right\|\right)\left\|x_{n}-x_{n+1}\right\| \\
& \leq \alpha_{n} M_{6}+\frac{M_{7}}{1-\beta_{n}}\left\|x_{n}-x_{n+1}\right\|,
\end{aligned}
$$

where $M_{7}>0$ is an appropriate constant. Therefore, by conditions (C1) and (C3) and Step 2, we derive from (3.34) that

$$
\left\|u_{n}-z_{n}\right\| \rightarrow 0 \text { as } n \rightarrow \infty
$$


Step 4. We show that $\lim _{n \rightarrow \infty}\left\|z_{n}-x_{n}\right\|=\lim _{n \rightarrow \infty}\left\|J_{\lambda_{n}}^{B} u_{n}-x_{n}\right\|=0$. First, from (3.31) and (3.33), we derive

$$
\begin{aligned}
\left\|x_{n+1}-p\right\|^{2} & \leq \beta_{n}\left\|x_{n}-p\right\|^{2}+\left(1-\beta_{n}\right)\left[\alpha_{n} M_{6}+\left(1-\alpha_{n} \tau\right)\left\|z_{t}-p\right\|^{2}\right] \\
& \leq \beta_{n}\left\|x_{n}-p\right\|^{2}+\left(1-\beta_{n}\right)\left[\alpha_{n} M_{6}+\left\|u_{n}-p\right\|^{2}-\left\|u_{n}-z_{n}\right\|^{2}\right] \\
& \leq \beta_{n}\left\|x_{n}-p\right\|^{2}+\left(1-\beta_{n}\right)\left[\alpha_{n} M_{6}+\left\|u_{n}-p\right\|^{2}\right] \\
& \leq\left\|x_{n}-p\right\|^{2}+\left(1-\beta_{n}\right)\left[\alpha_{n} M_{6}+\left\|x_{n}-p\right\|^{2}+\eta_{n}\left(L \eta_{n}-1\right)\left\|\left(J_{v_{n}}-I\right) A x_{n}\right\|^{2}\right] \\
& =\left\|x_{n}-p\right\|^{2}+\left(1-\beta_{n}\right) \alpha_{n} M_{6}+\left(1-\beta_{n}\right) \eta_{n}\left(L \eta_{n}-1\right)\left\|\left(R_{v_{n}}-I\right) A x_{n}\right\|^{2} .
\end{aligned}
$$

It follows that

$$
\begin{aligned}
\eta_{n}\left(1-L \eta_{n}\right)\left\|\left(R_{v_{n}}-I\right) A x_{n}\right\|^{2} & \leq \alpha_{n} M_{6}+\frac{1}{1-\beta_{n}}\left(\left\|x_{n}-p\right\|^{2}-\left\|x_{n+1}-p\right\|^{2}\right) \\
& \leq \alpha_{n} M_{6}+\frac{M_{7}}{1-\beta_{n}}\left\|x_{n}-x_{n+1}\right\| .
\end{aligned}
$$

Since $\left(1-L \eta_{n}\right)>0$ and $0<\eta \leq \liminf _{n \rightarrow \infty} \eta_{n}$, from conditions (C1) and (C3) and Step 2, it follows that

$$
\left\|\left(R_{v_{n}}-I\right) A x_{n}\right\| \rightarrow 0 \text { as } n \rightarrow \infty
$$

Next, using (2.1), (2.3), (3.7) and $\eta_{n} \in\left(0, \frac{1}{L}\right)$, we observe that

$$
\begin{aligned}
\left\|z_{n}-p\right\|^{2}= & \left\|J_{\lambda_{n}}^{B}\left(x_{n}+\eta_{n} A^{*}\left(R_{v_{n}}-I\right) A x_{n}\right)-p\right\|^{2} \\
= & \left\|J_{\lambda_{n}}^{B}\left(x_{n}+\eta_{t} A^{*}\left(R_{v_{n}}-I\right) A x_{n}\right)-J_{\lambda_{n}}^{B} p\right\|^{2} \\
\leq & \left\langle z_{n}-p, x_{n}+\eta_{n} A^{*}\left(R_{v_{n}}-I\right) A x_{n}-p\right\rangle \\
= & \frac{1}{2}\left\{\left\|z_{n}-p\right\|^{2}+\left\|x_{n}+\eta_{t} A^{*}\left(R_{v_{n}}-I\right) A x_{n}-p\right\|^{2}\right. \\
& \left.\quad-\left\|\left(z_{n}-p\right)-\left(x_{n}+\eta_{t} A^{*}\left(R_{v_{n}}-I\right) A x_{n}-p\right)\right\|^{2}\right\} \\
\leq & \frac{1}{2}\left\{\left\|z_{n}-p\right\|^{2}+\left\|x_{n}-p\right\|^{2}+\eta_{n}\left(L \eta_{n}-1\right)\left\|\left(R_{v_{n}}-I\right) A x_{n}\right\|^{2}\right. \\
& \left.\quad-\left\|\left(z_{n}-x_{n}\right)-\eta_{n} A^{*}\left(R_{v_{n}}-I\right) A x_{n}\right\|^{2}\right\} \\
\leq & \frac{1}{2}\left\{\left\|z_{n}-p\right\|^{2}+\left\|x_{n}-p\right\|^{2}-\left[\left\|z_{n}-x_{n}\right\|^{2}\right.\right. \\
\quad & \left.\left.+\eta_{n}^{2}\left\|A^{*}\left(R_{v_{n}}-I\right) A x_{n}\right\|^{2}-2 \eta_{n}\left\langle z_{n}-x_{n}, A^{*}\left(R_{v_{n}}-I\right) A x_{n}\right\rangle\right]\right\} \\
\leq & \frac{1}{2}\left\{\left\|z_{t}-p\right\|^{2}+\|x-p\|^{2}-\left\|z_{n}-x_{n}\right\|^{2}\right. \\
\quad & \left.\quad 2 \eta_{n}\left\|A\left(z_{n}-x_{n}\right)\right\|\left\|\left(R_{v_{n}}-I\right) A x_{n}\right\|\right\} .
\end{aligned}
$$

From (3.36), we obtain

$$
\left\|z_{n}-p\right\|^{2} \leq\left\|x_{n}-p\right\|^{2}-\left\|z_{n}-x_{n}\right\|^{2}+2 \eta_{n}\left\|A\left(z_{n}-x_{n}\right)\right\|\left\|\left(R_{v_{n}}-I\right) A x_{n}\right\| .
$$


Thus, from (2.4), (3.31) and (3.37), we derive

$$
\begin{aligned}
\left\|x_{n+1}-p\right\|^{2} \leq & \beta_{n}\left\|x_{n}-p\right\|^{2}+\left(1-\beta_{n}\right)\left[\alpha_{n}\left\|\gamma V x_{n}-\mu G p\right\|+\left(1-\alpha_{n} \tau\right)\left\|z_{n}-p\right\|\right]^{2} \\
\leq & \beta_{n}\left\|x_{n}-p\right\|^{2}+\left(1-\beta_{n}\right)\left(\alpha_{n} M_{6}+\left(1-\alpha_{n} \tau\right)\left\|z_{n}-p\right\|^{2}\right) \\
\leq & \beta_{n}\left\|x_{n}-p\right\|^{2}+\left(1-\beta_{n}\right)\left[\alpha_{n} M_{6}+\left\|z_{n}-p\right\|^{2}\right] \\
\leq & \beta_{n}\left\|x_{n}-p\right\|^{2}+\left(1-\beta_{n}\right)\left[\alpha_{n} M_{6}\right. \\
& \left.+\left(\left\|x_{n}-p\right\|^{2}-\left\|z_{n}-x_{n}\right\|^{2}+2 \eta_{n}\left\|A\left(z_{n}-x_{n}\right)\right\|\left\|\left(R_{v_{n}}-I\right) A x_{n}\right\|\right)\right] \\
= & \left\|x_{n}-p\right\|^{2}+\left(1-\beta_{n}\right) \alpha_{n} M_{6}-\left(1-\beta_{n}\right)\left\|z_{n}-x_{n}\right\|^{2} \\
& \left.+2\left(1-\beta_{n}\right) \eta_{n}\left\|A\left(z_{n}-x_{n}\right)\right\|\left\|\left(J_{v_{n}}^{B_{2}}-I\right) A x_{n}\right\|\right]
\end{aligned}
$$

which implies that

$$
\begin{aligned}
\left\|z_{n}-x_{n}\right\|^{2} \leq & \alpha_{n} M_{6}+2 \eta_{n}\left\|A\left(z_{n}-x_{n}\right)\right\|\left\|\left(R_{v_{n}}-I\right) A x_{n}\right\| \\
& +\frac{1}{1-\beta_{n}}\left(\left\|x_{n}-p\right\|^{2}-\left\|x_{n+1}-p\right\|^{2}\right) \\
\leq & \alpha_{n} M_{6}+2 \eta_{n}\left\|A\left(z_{n}-x_{n}\right)\right\|\left\|\left(R_{v_{n}}-I\right) A x_{n}\right\| \\
& +\frac{1}{1-\beta_{n}}\left(\left\|x_{n}-p\right\|+\left\|x_{n+1}-p\right\|\right)\left\|x_{n}-x_{n+1}\right\| \\
\leq & \alpha_{n} M_{6}+2 \eta_{n}\left\|A\left(z_{n}-x_{n}\right)\right\|\left\|\left(R_{v_{n}}-I\right) A x_{n}\right\| \\
& +\frac{1}{1-\beta_{n}}\left\|x_{n}-x_{n+1}\right\| M_{7} .
\end{aligned}
$$

Thus, from conditions (C1) and (C3), Step 2, (3.35) and (3.38), it follows that

$$
\left\|z_{n}-x_{n}\right\| \rightarrow 0 \text { as } n \rightarrow \infty \text {. }
$$

Step 5. We show that $\lim _{n \rightarrow \infty}\left\|x_{n}-u_{n}\right\|=0$. In fact, by Steps 3 and 4 , we have

$$
\left\|x_{n}-u_{n}\right\| \leq\left\|x_{n}-z_{n}\right\|+\left\|z_{n}-u_{n}\right\| \rightarrow 0 \text { as } n \rightarrow \infty .
$$

Step 6. We show that $\lim _{n \rightarrow \infty}\left\|x_{n}-y_{n}\right\|=0$. In fact, since

$$
\begin{aligned}
\left\|x_{n}-y_{n}\right\| & =\left\|x_{n}-\left(\alpha_{n} \gamma V x_{n}+\left(I-\alpha_{n} \mu G\right) J_{\lambda_{n}}^{B_{1}} u_{n}\right)\right\| \\
& \leq \alpha_{n}\left\|\mu G x_{n}-\gamma V x_{n}\right\|+\left\|\left(I-\alpha_{n} \mu G\right) x_{n}-\left(I-\alpha_{n} \mu G\right) z_{n}\right\| \\
& \leq \alpha_{n} M_{8}+\left(1-\alpha_{n} \tau\right)\left\|x_{n}-z_{n}\right\| \\
& \leq \alpha_{n} M_{8}+\left\|x_{n}-z_{n}\right\|,
\end{aligned}
$$

where $M_{8}>0$ is an appropriate constant, by condition (C1) and Step 4, we obtain

$$
\left\|x_{n}-y_{n}\right\| \rightarrow 0 \text { as } n \rightarrow \infty \text {. }
$$

Step 7. We show that $\lim _{n \rightarrow \infty}\left\|y_{n}-T_{r_{n}} y_{n}\right\|=\lim _{n \rightarrow \infty}\left\|y_{n}-w_{n}\right\|=0$. Indeed, from (3.30) and Step 6, it follows that

$$
\left\|y_{n}-T_{r_{n}} y_{n}\right\| \leq\left\|y_{n}-x_{n}\right\|+\left\|x_{n}-w_{n}\right\| \rightarrow 0 \text { as } n \rightarrow \infty .
$$

Step 8. We show that

$$
\limsup _{n \rightarrow \infty}\left\langle(\gamma V-\mu G) q, y_{n}-q\right\rangle \leq 0
$$


For this purpose, we can choose a subsequence $\left\{y_{n_{i}}\right\}$ of $\left\{y_{n}\right\}$ such that

$$
\lim _{i \rightarrow \infty}\left\langle(\gamma V-\mu G) q, y_{n_{i}}-q\right\rangle=\limsup _{n \rightarrow \infty}\left\langle(\gamma V-\mu G) q, y_{n}-q\right\rangle .
$$

Since $\left\{y_{n_{i}}\right\}$ is bounded, there exists a subsequence $\left\{y_{n_{i_{j}}}\right\}$ of $\left\{y_{n_{i}}\right\}$, which converges weakly to some point $z$. Without loss of generality, we can assume that $y_{n_{i}} \rightarrow z$. First of all, we show $z \in \Omega$. To this end, we divide its proof into three steps.

(i) We prove that $z \in F i x(T)$. To show this, we put $w_{n}=T_{r_{n}} y_{n}$. Then, by Lemma 2.6, we have

$$
\left\langle y-w_{n}, T w_{n}\right\rangle-\frac{1}{r_{n}}\left\langle y-w_{n},\left(1+r_{n}\right) w_{n}-y_{n}\right\rangle \leq 0, \quad \forall y \in H_{1} .
$$

Put $v_{\varepsilon}=\varepsilon v+(1-\varepsilon) z$ for $\varepsilon \in(0,1]$ and $v \in H_{1}$. Then $v_{\varepsilon} \in H_{1}$, and from (3.39) and pseudocontractivity of $T$, it follows that

$$
\begin{aligned}
\left\langle w_{n}-v_{\varepsilon}, T v_{\varepsilon}\right\rangle \geq\left\langle w_{n}-v_{\varepsilon}, T v_{\varepsilon}\right\rangle+\left\langle v_{\varepsilon}-w_{n}, T w_{n}\right\rangle & -\frac{1}{r_{n}}\left\langle v_{\varepsilon}-w_{n},\left(1+r_{n}\right) w_{n}-y_{n}\right\rangle \\
= & -\left\langle v_{\varepsilon}-w_{n}, T v_{\varepsilon}-T w_{n}\right\rangle-\frac{1}{r_{n}}\left\langle v_{\varepsilon}-w_{n}, w_{n}-y_{n}\right\rangle \\
& -\left\langle v_{\varepsilon}-w_{n}, w_{n}\right\rangle \\
\geq & -\left\|v_{\varepsilon}-w_{n}\right\|^{2}-\frac{1}{r_{n}}\left\langle v_{\varepsilon}-w_{n}, w_{n}-y_{n}\right\rangle-\left\langle v_{\varepsilon}-w_{n}, w_{n}\right\rangle \\
= & -\left\langle v_{\varepsilon}-w_{n}, v_{\varepsilon}\right\rangle-\left\langle v_{\varepsilon}-w_{n}, \frac{w_{n}-y_{n}}{r_{n}}\right\rangle .
\end{aligned}
$$

Since $\left\{y_{n}\right\}$ and $\left\{w_{n}\right\}$ have the same asymptotical behavior (due to Step 7), $w_{n_{i}} \rightarrow z$ as $i \rightarrow \infty$. Also, by Step 7, we have

$$
\frac{\left\|w_{n}-y_{n}\right\|}{r_{n}} \leq \frac{\left\|w_{n}-y_{n}\right\|}{r} \rightarrow 0 .
$$

So, replacing $n$ by $n_{i}$ and letting $i \rightarrow \infty$, we derive from (3.40) that

$$
\left\langle z-v_{\varepsilon}, T v_{\varepsilon}\right\rangle \geq\left\langle z-v_{\varepsilon}, v_{\varepsilon}\right\rangle
$$

and

$$
-\left\langle v-z, T v_{\varepsilon}\right\rangle \geq-\left\langle v-z, v_{\varepsilon}\right\rangle, \quad \forall v \in H_{1} .
$$

Letting $\varepsilon \rightarrow 0$ and using the fact that $T$ is continuous, we obtain

$$
-\langle v-z, T z\rangle \geq-\langle v-z, z\rangle, \quad \forall v \in H_{1} .
$$

Let $v=T z$ in (3.41). Then we have $z=T z$, that is, $z \in F i x(T)$.

(ii) We prove that $z \in B^{-1} 0$. To this end, let $z_{n}=J_{\lambda_{n}}^{B_{1}} u_{n}$. Then it follows that

$$
u_{n} \in\left(I+\lambda_{n} B\right) z_{n}, \text { that is, } \frac{u_{n}-x_{n}}{\lambda_{n}} \in B z_{n} .
$$

Since $B$ is monotone, we know that, for any $v \in B u$,

$$
\left\langle z_{n}-u, \frac{u_{n}-z_{n}}{\lambda_{n}}-v\right\rangle \geq 0 .
$$


Since

$$
\frac{\left\|u_{n}-z_{n}\right\|}{\lambda_{n}} \leq \frac{\left\|u_{n}-J_{\lambda_{n}}^{B} u_{n}\right\|}{\lambda} \rightarrow 0
$$

as $n \rightarrow \infty$ by Step 3 and $\left\|x_{n}-u_{n}\right\| \rightarrow 0$ as $n \rightarrow \infty$ by Step 5, we have $u_{n_{i}} \rightarrow z$ and $z_{n_{i}} \rightarrow z$ as $i \rightarrow \infty$ by Steps 3, 5 and 6. By replacing $n$ by $n_{i}$ in (3.42) and letting $i \rightarrow \infty$, we have $\langle z-u,-v\rangle \geq 0$. Since $B$ is maximal monotone, we get $0 \in B z$, that is, $z \in B^{-1} 0$.

(iii) We prove that $A q \in F i x(R)$. In fact, since $\left\{x_{n}\right\}$ and $\left\{u_{n}\right\}$ have the same asymptotical behavior (due to Step 5), $\left\{A x_{n_{i}}\right\}$ converges weakly to $A z$. Again, let $\hat{v}>0$. Then, using (3.19) and (3.35), we estimate

$$
\left\|R_{v_{n_{i}}}\left(A x_{n_{i}}\right)-R_{\hat{v}}\left(A x_{n_{i}}\right)\right\| \leq \frac{\left|v_{n_{i}}-\hat{v}\right|}{v}\left\|\left(R_{v_{n_{i}}}-I\right) A x_{n_{i}}\right\| \rightarrow 0 \text { as } i \rightarrow \infty
$$

Hence, from (3.43), it follows that

$$
\lim _{i \rightarrow \infty}\left\|\left(R_{\hat{v}}-I\right) A x_{n_{i}}\right\|=\lim _{i \rightarrow \infty}\left\|\left(J_{v_{n_{i}}}^{B_{2}}-I\right) A x_{n_{i}}\right\|=0 .
$$

Since $R_{\hat{v}}$ is nonexpansive, by (3.44) and Lemma 2.9, we obtain the $A z=R_{\hat{v}}(A z)$, that is, $A z \in$ $F i x\left(R_{\hat{v}}\right)=F i x(R)$. This means that $z \in A^{-1}(F i x(R))$. This along with (i) and (ii) obtains $z \in \Omega$. Thus

$$
\begin{aligned}
\limsup _{n \rightarrow \infty}\left\langle(\gamma V-\mu G) q, y_{n}-q\right\rangle & =\lim _{i \rightarrow \infty}\left\langle(\gamma V-\mu G) q, y_{n_{i}}-q\right\rangle \\
& =\langle(\gamma V-\mu G) q, z-q\rangle \leq 0
\end{aligned}
$$

Step 9. We show that $\lim _{n \rightarrow \infty}\left\|x_{n}-q\right\|=0$. Indeed, from Lemma 2.3, we derive

$$
\begin{aligned}
\left\|y_{n}-q\right\|^{2}= & \left\|\alpha_{n} \gamma V x_{n}+\left(I-\alpha_{n} \mu G\right) J_{\lambda_{n}}^{B} u_{n}-q\right\|^{2} \\
= & \| \alpha_{n}\left(\gamma V x_{n}-\gamma V q\right)+\alpha_{n}(\gamma V q-\mu G q) \\
& \quad+\left(I-\alpha_{n} \mu G\right) J_{\lambda_{n}}^{B} u_{n}-\left(I-\alpha_{n} \mu G\right) q \|^{2} \\
\leq & \left\|\alpha_{n}\left(\gamma V x_{n}-\gamma V q\right)+\left(I-\alpha_{n} \mu G\right) J_{\lambda_{n}}^{B} u_{n}-\left(I-\alpha_{n} \mu G\right) q\right\|^{2} \\
& \quad+2 \alpha_{n}\left\langle\gamma V q-\mu G q, y_{n}-q\right\rangle \\
\leq & \left(\alpha_{n} \gamma l\left\|x_{n}-q\right\|+\left(1-\alpha_{n} \tau\right)\left\|x_{n}-q\right\|\right)^{2} \\
& +2 \alpha_{n}\left\langle\gamma V q-\mu G q, y_{n}-q\right\rangle \\
= & \left(\left(1-(\tau-\gamma l) \alpha_{n}\right)\left\|x_{n}-q\right\|\right)^{2}+2 \alpha_{n}\left\langle\gamma V q-\mu G q, y_{n}-q\right\rangle .
\end{aligned}
$$


Thus, by (2.4), (3.1) and (3.45), we obtain

$$
\begin{aligned}
\left\|x_{n+1}-q\right\|^{2} \leq & \beta_{n}\left\|x_{n}-q\right\|^{2}+\left(1-\beta_{n}\right)\left\|T_{r_{n}} y_{n}-q\right\|^{2} \\
\leq & \beta_{n}\left\|x_{n}-q\right\|^{2}+\left(1-\beta_{n}\right)\left\|y_{n}-q\right\|^{2} \\
\leq & \beta_{n}\left\|x_{n}-q\right\|^{2}+\left(1-\beta_{n}\right)\left(\left(1-(\tau-\gamma l) \alpha_{n}\right)\left\|x_{n}-q\right\|\right)^{2} \\
& +2\left(1-\beta_{n}\right) \alpha_{n}\left\langle\gamma V q-\mu G q, y_{n}-q\right\rangle \\
\leq & \beta_{n}\left\|x_{n}-q\right\|^{2}+\left(1-\beta_{n}\right)\left(1-(\tau-\gamma l) \alpha_{n}\right)\left\|x_{n}-q\right\|^{2} \\
& +2\left(1-\beta_{n}\right) \alpha_{n}\left\langle\gamma V q-\mu G q, y_{n}-q\right\rangle \\
= & \left(1-\left(1-\beta_{n}\right)(\tau-\gamma l) \alpha_{n}\right)\left\|x_{n}-q\right\|^{2} \\
& +2\left(1-\beta_{n}\right)(\tau-\gamma l) \alpha_{n} \frac{\left\langle\gamma V q-\mu G q, y_{n}-q\right\rangle}{\tau-\gamma l} \\
= & \left(1-\xi_{n}\right)\left\|x_{n}-q\right\|^{2}+\xi_{n} \delta_{n},
\end{aligned}
$$

where

$$
\xi_{n}=\left(1-\beta_{n}\right)(\tau-\gamma l) \alpha_{n}
$$

and

$$
\delta_{n}=\frac{2\left\langle\gamma V q-\mu G, y_{n}-q\right\rangle}{\tau-\gamma l} .
$$

From conditions (C1), (C2) and (C3) and Step 8, it is easy to see that $\xi_{n} \rightarrow 0, \sum_{n=1}^{\infty} \xi_{n}=\infty$ and $\limsup _{n \rightarrow \infty} \delta_{n} \leq 0$. Hence, by Lemma 2.5, we conclude $\lim _{n \rightarrow \infty}\left\|x_{n}-q\right\|=0$. This completes the proof.

By taking $V \equiv 0, G \equiv I, \mu=1$ in Theorem 3.1, we obtain the following result.

Corollary 3.1. Let the sequence $\left\{x_{n}\right\}$ be generated by

$$
\left\{\begin{aligned}
z_{n} & =J_{r_{n}}^{B}\left(x_{n}+\eta_{n} A^{*}\left(R_{v_{n}}-I\right) A x_{n}\right), \\
x_{n+1} & =\beta_{n} x_{n}+\left(1-\beta_{n}\right) T_{r_{n}}\left(\left(1-\alpha_{n}\right) z_{n}\right), \quad n \geq 0 .
\end{aligned}\right.
$$

Let $\left\{\alpha_{n}\right\},\left\{\beta_{n}\right\} \subset(0,1),\left\{r_{n}\right\},\left\{\lambda_{n}\right\},\left\{v_{n}\right\} \subset(0, \infty)$ and $\left\{\eta_{n}\right\} \subset\left(0, \frac{1}{L}\right)$ satisfy the conditions (C1), (C2), (C3), (C4), (C5), (C6) and (C7) in Theorem 3.1. Then $\left\{x_{n}\right\}$ converges strongly to a point $q \in \Omega$, which is the minimum-norm element of $\Omega$.

Proof. From (3.2) with $V \equiv 0, G \equiv I$ and $\mu=1$, we derive

$$
0 \leq\langle q, p-q\rangle, \quad \forall p \in \Omega .
$$

This obviously implies that

$$
\|q\|^{2} \leq\langle p, q\rangle \leq\|p\|\|q\|, \quad \forall p \in \Omega .
$$

It turns out that $\|q\| \leq\|p\|$ for all $p \in \Omega$. Therefore, $q$ is the minimum-norm point of $\Omega$.

If in Theorem 3.1, we take $T \equiv I$, identity mapping on $H_{1}$, then we obtain the following result. 
Corollary 3.2. Let the sequence $\left\{x_{n}\right\}$ be generated by

$$
\left\{\begin{aligned}
z_{n} & =J_{r_{n}}^{B}\left(x_{n}+\eta_{n} A^{*}\left(R_{v_{n}}-I\right) A x_{n}\right), \\
x_{n+1} & =\beta_{n} x_{n}+\left(1-\beta_{n}\right)\left(\alpha_{n} \gamma V x_{n}+\left(I-\alpha_{n} \mu G\right) z_{n}\right), \quad n \geq 0 .
\end{aligned}\right.
$$

Let $\left\{\alpha_{n}\right\},\left\{\beta_{n}\right\} \subset(0,1),\left\{\lambda_{n}\right\},\left\{v_{n}\right\} \subset(0, \infty)$ and $\left\{\eta_{n}\right\} \subset\left(0, \frac{1}{L}\right)$ satisfy the conditions (C1), (C2), (C3), (C5), (C6) and (C7) in Theorem 3.1. Then $\left\{x_{n}\right\}$ converges strongly to a point $q \in B^{-1} 0 \cap A^{-1}(F i x(R))$, which is the unique solution of the following variational inequality:

$$
\langle(\mu G-\gamma V) q, p-q\rangle \geq 0, \quad \forall p \in B^{-1} 0 \cap A^{-1}(F i x(R)) .
$$

By taking $V \equiv 0, G \equiv I, \mu=1$ in Corollary 3.2, we obtain the following result.

Corollary 3.3. Let the sequence $\left\{x_{n}\right\}$ be generated by

$$
\left\{\begin{aligned}
z_{n} & =J_{r_{n}}^{B}\left(x_{n}+\eta_{n} A^{*}\left(R_{v_{n}}-I\right) A x_{n}\right), \\
x_{n+1} & =\beta_{n} x_{n}+\left(1-\beta_{n}\right)\left(1-\alpha_{n}\right) z_{n}, \quad n \geq 0 .
\end{aligned}\right.
$$

Let $\left\{\alpha_{n}\right\},\left\{\beta_{n}\right\} \subset(0,1),\left\{r_{n}\right\},\left\{\lambda_{n}\right\},\left\{v_{n}\right\} \subset(0, \infty)$ and $\left\{\eta_{n}\right\} \subset\left(0, \frac{1}{L}\right)$ satisfy the conditions $(\mathrm{C} 1),(\mathrm{C} 2),(\mathrm{C} 3),(\mathrm{C} 5),(\mathrm{C} 6)$ and (C7) in Theorem 3.1. Then $\left\{x_{n}\right\}$ converges strongly to a point $q \in B^{-1} 0 \cap A^{-1}(F i x(R))$, which is the minimum-norm element of $B^{-1} 0 \cap A^{-1}(F i x(R))$.

Remark 3.1. 1) It is worth pointing out that our general iterative algorithm is a new one, which is different from those announced by several authors; see, e.g., [14, 20, 24] and the references therein. In particular, we use the variable parameters $r_{n}, \lambda_{n}, v_{n}$, and $\eta_{n}$ in comparison with the corresponding iterative algorithms in $[14,20,24]$ and the references therein.

2) Our general iterative algorithm (3.1) is very different from the associated one in [24] (i.e., (1.4) in 1. Introduction) because the first iterative step $z_{n}=J_{\lambda_{n}}^{B}\left(x_{n}+\lambda_{n} A^{*}(S-\right.$ I)A $\left.x_{n}\right)$ in [24] is replaced by the first step $z_{n}=J_{\lambda_{n}}^{B}\left(x_{n}+\eta_{n} A^{*}\left(R_{v_{n}}-I\right) A x_{n}\right)$ in our iterative algorithm (3.1), and the second iterative steps $x_{n+1}=\beta_{n} x_{n}+\left(1-\beta_{n}\right) U z_{n}$ in [24] is replaced by the second step $x_{n+1}=\beta_{n} x_{n}+\left(1-\beta_{n}\right) T_{r_{n}}\left(\alpha_{n} \gamma V x_{n}+\left(I-\alpha_{n} \mu G\right) z_{n}\right)$ in our iterative algorithm (3.1).

3) Theorem 3.1 improves and develops the corresponding results in [24] in following aspects:

(a) The nonexpansive mapping $S$ in [24] is extended to the case of the continuous pseudocontractive mapping $R$.

(b) A generalized hybrid mapping $U$ is replaced with the continuous pseudocontractive mapping $T$.

(c) In order to establish strong convergence in place of weak convergence, a Lipschizian mapping $V$ with a constant $l \geq 0$ and a $\rho$-Lipschitzian and $\eta$-strongly monotone mapping $G$ are utilized in comparison with [24].

4) Corollary 3.1 is a new result for finding a minimum norm point of $B^{-1} 0 \cap F i x(T)$ $\cap A^{-1}(\operatorname{Fix}(R))$.

5) Corollary 3.2 and Corollary3.3 are also new results for finding a point of $B^{-1} 0 \cap$ $A^{-1}(F i x(R))$ and a minimum norm point of $B^{-1} 0 \cap A^{-1}(F i x(R))$, respectively. 
6) As a application, if $J_{v_{n}}^{F}=R_{v_{n}}$ in Theorem 3.1, where $J_{v_{n}}^{F}=\left(I+v_{n} F\right)^{-1}: H_{2} \rightarrow \operatorname{dom}(F)$ is the resolvent of the maximal monotone operator $F: H_{2} \rightarrow 2^{H_{2}}$, then we can propose a general explicit iterative algorithm for finding a solution in $\left.B^{-1} 0 \cap A^{-1}\left(F^{-1} 0\right)\right) \cap$ Fix $(T)$, which is a common solution of the split variational inclusion problem and the fixed point problem for a pseudocontractive mapping. This iterative algorithm includes, as special cases, the corresponding those in $[14,20]$. Even though this case, the condition $\sum_{n=1}^{\infty}\left|\alpha_{n+1}-\alpha_{n}\right|<\infty$ on the control parameter $\left\{\alpha_{n}\right\}$ in $[14,20]$ was dispensed.

\section{Acknowledgments}

This research was supported by the Basic Science Research Program through the National Research Foundation of Korea (NRF) funded by the Ministry of Education, Science and Technology (2018R1D1A1B07045718).

\section{REFERENCES}

[1] R.P. Agarwal, D. O’Regan, D.R. Sahu, Fixed Point Theory for Lipschitzian-type Mappings with Applications, Springer, New York, (2009).

[2] C. Byrne, Iterative oblique projection onto convex sets and the split feasibility problem, Inverse Prob. 18 (2002), 441-453.

[3] C. Byrne, A unified treatment for some iterative algorithms in signal processing and image reconstruction, Inverse Probl. 20 (2004), 103-120.

[4] C. Byrne, Y. Censor, A. Gibali, S. Reich, Weak and strong convergence of algorithms for the split common null point problem, J. Nonlinear Convex Anal. 13 (2012), 759-775.

[5] F.E. Browder, W.V. Petryshn, Construction of fixed points of nonlinear mappings in Hilbert space, J. Math. Anal. Appl. 20 (1967), 197-228.

[6] Y. Censor, T. Bortfeld, B. Martin, A. Tronimov, A unified approach for inversion problems in intensity modulated radiation therapy, Phys. Med. Biol. 51 (2003), 2353-2365.

[7] Y. Censor, T. Elfving, A multiprojection algorithm using Bregman projections in a product space, Numer. Algo. 8 (1994), 221-239.

[8] P.L. Combettes, The convex feasibility problem in image recovery, In: P. Hawkes, (ed.) Advances inImaging and Electron Physics, vol. 95, pp. 155-270, Academic Press, New York, 1996.

[9] G. Crombez, A hierarchical presentation of operators with fixed points on Hilbert spaces, Numer. Funct. Anal. Optim. 27 (2006), 259-277.

[10] K. Goebel, S. Reich, Topics on Metric Fixed Point Theory, Cambridge University Press, Cambridge, 1990.

[11] H. Iiduka, W. Takahashi, Strong convergence theorems for nonexpansive mappings and inverse-strongly monotone mappings, Nonlinear Anal. 61 (2005), 341-350.

[12] J.S. Jung, Iterative algorithms based on the hybrid steepest descent method for the split feasibility problem, J. Nonlinear Sci. Appl. 9 (2016), 4214-4225.

[13] J.S. Jung, Hybrid iterative algorithms for the split common fixed point problems, J. Nonlinear Sci. Appl. 10 (2017), 2214-2228.

[14] K. Kazmi, S. Rizvi, An iterative method for split variational inclusion problem and fixed point problem for a nonexpansive mapping, Optim. Lett. 8 (2014), 1113-1124

[15] P. Kocourek, W. Takahashi, J.C. Yao, Fixed point theorems and weak convergences theorems for generalized hybrid mappings in Hilbert spaces, Taiwan J. Math. 14 (2010), 2497-2511.

[16] F. Kosaka, W. Takahashi, Existence and approximation of fixed points of firmly nonexpansive-type mapping in Banach spaces, SIAM J. Optim. 19 (2008), 824-835.

[17] F. Kosaka, W. Takahashi, Fixed point theorems for a class of nonexpansive mappings related to maximal monotone operators in Banach spaces, Arch. Math. (Basel) 91 (2008), 166-177.

[18] A. Moudafi, The split common fixed point problem for demicontractive mappings, Inverse Probl. 26 (2010), 0550070 . 
[19] A. Moudafi, Split monotone variational inclusions, J. Optim. Theory Appl. 150 (2011), 275-283.

[20] K. Sitthithakerngkiet, J. Deepho, P. Kumam, A hybrid viscosity algorithm via modify the hybrid steepest descent method for solving the split variarional inclusion in image reconstruction and fixed point problems, Appl. Math. Comput. 250 (2015), 986-1001.

[21] T. Suzuki, Strong convergence of Krasnoselskii and Mann's type sequences for one parameter nonexpansive semigroups without Bochner integral, J. Math. Anal. Appl. 305 (2005), 227-239.

[22] W. Takahashi, Introduction to Nonlinear and Convex Analysis, Yokohama Publishers, Yokohama, 2009.

[23] W. Takahashi, Fixed point theorems for new nonlinear mappings in a Hilbert space, J. Nonlinear Convex Anal. 11 (2010), 79-88.

[24] W. Takahashi, H. K. Xu, J. C. Yao, Iterative methods for generalized split feasibility problems in Hilbert spaces, Set-Valued Var. Anal. 23 (2015), 205-221.

[25] G. Wu, S. Luo, Adaptive fixed-point iterative shringkage/threshoding algorithm for MR imaging reconstruction using compressed sensing, Magn. Reson. Imaging 32 (2014), 372-378.

[26] H. K. Xu, Iterative algorithms for nonlinear operators, J. London Math. Soc. 66 (2002), 240-256.

[27] I. Yamada, The hybrid steepest descent method for the variational inequality of the intersection of fixed point sets of nonexpansive mappings, Inherently Parallel Algorithm for Feasibility and Optimization, and Their Applications, North-Holland, Amsterdam, Stud. Comput. Math. 8 (2001), 473-504.

[28] H. Zegeye, An iterative approximation method for a common fixed point of two pseudocontractive mappings, ISRN Math. Anal. 2011 (2011), 621901. 